\title{
Kantrovich Type Generalization of Meyer-König and Zeller Operators via Generating Functions
}

\author{
Ali Olgun, H. Gül İnce and Fatma Taşdelen
}

\begin{abstract}
In the present paper, we study a Kantorovich type generalization of Meyer-König and Zeller type operators via generating functions. Using Korovkin type theorem we first give approximation properties of these operators defined on the space $C[0, A], 0<A<1$. Secondly, we compute the rate of convergence of these operators by means of the modulus of continuity and the elements of the modified Lipschitz class. Finally, we give an $r$-th order generalization of these operators in the sense of Kirov and Popova and we obtain approximation properties of them.
\end{abstract}

\section{Introduction}

For a function $f$ on $[0,1)$, the Meyer -König and Zeller operators ( MKZ ) (see[11]) are given by

$$
M_{n}(f ; x)=(1-x)^{n+1} \sum_{k=0}^{\infty} f\left(\frac{k}{n+k+1}\right)\left(\begin{array}{c}
n+k \\
k
\end{array}\right) x^{k}, n \in \mathbb{N}
$$

Where $x \in[0,1)$. The approximation properties of the operators have been studied by Lupaş and Müller [12]. A slight modification of these operatos, called Bernstein power series, was introduced by Cheney and Sharma[3]

Key Words: Positive Linear operators,Kantorovich-type operators, Meyer-König and Zeller operators, Modulus of contiunity, Modified Lipschitz class, r-th order generalization. 2010 Mathematics Subject Classification: Primary 41A25; Secondary 41A36.

Received: April, 2011.

Revised: April, 2011.

Accepted: February, 2012. 
and Khan[8] obtained the rate of convergence of Bernstein power series for functions of bounded variation. The Meyer-König and Zeller operators were also generalized in [4] by Doğru. A Stancu type generalization of the operators (1) have been studied by Agratini [2]. Doğru and Özalp [5] studied a Kantorovich type generalization of the operators. Using statistically convergence, a Kantorovich type generalization of Agratini's operators have been studied by Doğru, Duman and Orhan in [6]. Furthermore Altın, Doğru and Taşdelen [1] introduced a generalization of the operators (1) via linear generating functions as follows:

$$
L_{n}(f ; x)=\frac{1}{h_{n}(x, t)} \sum_{k=0}^{\infty} f\left(\frac{a_{k, n}}{a_{k, n}+b_{n}}\right) \Gamma_{k, n}(t) x^{k}
$$

where $0<\frac{a_{k, n}}{a_{k, n}+b_{n}}<A, A \in(0,1)$, and $\left\{h_{n}(x, t)\right\}_{n \in \mathbb{N}}$ is the generating function for the sequence of function $\left\{\Gamma_{k, n}(t)\right\}_{n \in \mathbb{N}_{0}}$ in the form

$$
h_{n}(x, t)=\sum_{k=0}^{\infty} \Gamma_{k, n}(t) x^{k}, \quad t \in I
$$

where $I$ is any subinterval of $\mathbb{R}, \mathbb{N}_{0}=\mathbb{N} \cup\{0\}$. The Authors studied approximation properties of the operators (2) under the following conditions (see [1]):

i) $h_{n}(x, t)=(1-x) h_{n+1}(x, t)$

ii) $b_{n} \Gamma_{k, n+1}(t)=a_{k+1, n} \Gamma_{k+1, n}(t)$

iii) $b_{n} \rightarrow \infty, \frac{b_{n+1}}{b_{n}} \rightarrow 1, b_{n} \neq 0$, for all $n \in \mathbb{N}$

iv) $\Gamma_{k, n}(t) \geq 0$, for all $I \subset \mathbb{R}$

v) $a_{k+1, n}=a_{k, n+1}+\varphi_{n},\left|\varphi_{n}\right| \leq m<\infty, a_{0, n}=0$

On the other hand, Korovkin type theorems (see [10]) on some general Lipschitz type maximal functions spaces were given by Gadjiev and Çakar [7] and Doğru [4], including the test functions $\left(\frac{x}{1-x}\right)^{v}$ and $\left(\frac{a_{n} x}{1+a_{n} x}\right)^{v}(v=0,1,2)$, respectively. The space of Lipschitz type maximal functions was defined by Lenze in [11]. Altın, Doğru and Taşdelen [1] obtained a Korovkin type theorem using the test functions $\left(\frac{x}{1-x}\right)^{v} \quad(v=0,1,2)$, for the investigation of the approximation properties of the operators (2). They used the nodes $s=\frac{a_{k, n}}{a_{k, n}+b_{n}}$, with $\frac{s}{1-s}=\frac{a_{k, n}}{b_{n}}$.

We known from [1] that the operators $L_{n}$ given by (2) satisfy the the following equalities:

$$
\begin{gathered}
L_{n}(1 ; x)=1, \\
L_{n}(\theta(s) ; x)=\theta(x),
\end{gathered}
$$




$$
L_{n}\left(\theta^{2}(s) ; x\right)=(\theta(x))^{2} \frac{b_{n+1}}{b_{n}}+\frac{\varphi_{n}}{b_{n}} \theta(x),
$$

where $\theta(s)=\frac{s}{1-s}, \quad x \in[0, A], 0<A<1, t \in I, n \in \mathbb{N}$.

Throughout our present investigation, by $\|$.$\| , we denote the usual supre-$ mum norm on the space $C[0, A]$ which is the space of all continuous functions on $[0, A]$.

Clearly by (4), (5) and (6) it follows that

$$
\lim _{n \rightarrow \infty}\left\|L_{n}\left(\theta^{v}\right)-\theta^{v}\right\|=0 ; v=0,1,2 .
$$

The objective of this paper is to investigate the Kantorovich type generalization of the operators $L_{n}$. In the next section, we present the integral type extension of $L_{n}$. In section 3, we study the rate of convergence of our operators by means of the modulus of continuity and the elements of the modified Lipschitz class. Finally, in section 4 , we give an $r$-th order generalization for these operators and approximation properties of them.

\section{Construction of the Kantorovich-type Operators}

Now, we set

$$
\theta(s):=\frac{s}{1-s} .
$$

In this section, we construct a Kantorovich type generalization of MKZ type operators via generating functions and we investigate the approximation properties of these operators with the aid of the test functions $\theta^{v}, v=0,1,2$. We should note here that in the sequel we shall use the letter $\theta$ for the test function $\theta(s)$.

Let $\omega$ be the modulus of continuity of $f$ defined as

$$
\omega(f, \delta):=\sup \{|f(s)-f(x)| ; s, x \in[0, A],|s-x|<\delta\}
$$

for $f \in C[0, A]$.

Let $\omega=\omega(t)$ be arbitrary modulus of continuity defined on $[0, A]$, which satisfies the following conditions [14]

a) $\omega$ is non- decreasing,

b) $\omega\left(\delta_{1}+\delta_{2}\right) \leq \omega\left(\delta_{1}\right)+\omega\left(\delta_{2}\right)$ for $\delta_{1}, \delta_{2} \in[0, A]$

c) $\lim _{\delta \rightarrow 0^{+}} \omega(\delta)=0$.

Inspired of the well-known $H_{\omega}$ class (see for ex. p 108 of [16]), we now define the following $W_{\omega}$ class. We say that a function $f \in C[0, A]$ belongs to the class $W_{\omega}[0, A]$ if $\left|f\left(t_{1}\right)-f\left(t_{2}\right)\right| \leq \omega\left(\left|\frac{t_{1}}{1-t_{1}}-\frac{t_{2}}{1-t_{2}}\right|\right)$ for all $t_{1}, t_{2} \in[0, A]$. Clearly, if $f \in W_{\omega}[0, A]$, then $\omega(f, \delta) \leq \omega(\delta)$ for all $\delta \in[0, A]$. Now, fix 
$\omega(t)=t$, an example of the function belonging to the $W_{\omega}$ class can be given by $f(x)=\frac{1}{1-x}$. Indeed, $|f(s)-f(x)| \leq \frac{|s-x|}{(1-x)(1-s)}=\omega\left(\left|\frac{s}{1-s}-\frac{x}{1-x}\right|\right)$.

Let $W_{\omega}$ be the space of real valued functions $f \in C[0, A]$ satisfying

$$
|f(s)-f(x)| \leq \omega\left(\left|\frac{s}{1-s}-\frac{x}{1-x}\right|\right), \text { for any } x, s \in[0, A] .
$$

It can easily be obtained that $\omega$ satisfies

$$
\omega(n \delta) \leq n \omega(\delta), \quad n \in \mathbb{N}
$$

and from the property $b$ ) of the modulus of continuity $\omega$ we have

$$
\omega(\lambda \delta) \leq \omega((1+\|\lambda\|) \delta) \leq(1+\lambda) \omega(\delta), \quad \lambda>0,
$$

where $\|\lambda\|$ is the greatest integer of $\lambda$.

Let us assume that the properties $(i)-(v)$ are satisfied. We also assume that $\left\{b_{n}\right\}$ is a positive increasing sequence.

Now we modify the operators $L_{n}$ by replacing $f\left(\frac{a_{k, n}}{a_{k, n}+b_{n}}\right)$ in (2) by an integral mean of $f$ over an interval $I_{k, n}=\left[a_{k, n}, a_{k, n}+c_{k, n}\right]$ as follows:

$$
L_{n}^{*}(f(s) ; x)=L_{n}^{*}(f ; x)=\frac{1}{h_{n}(x, t)} \sum_{k=0}^{\infty} \frac{\Gamma_{k, n}(t)}{c_{k, n}} x^{k} \int_{a_{k, n}}^{a_{k, n}+c_{k, n}} f\left(\frac{\xi}{\xi+b_{n}}\right) d \xi
$$

where $f$ is an integrable function on the interval $(0,1)$ and $\left\{c_{k, n}\right\}$ is a sequence such that

$$
0<c_{k, n} \leq 1, \text { for every } k \in \mathbb{N} .
$$

Clearly, $L_{n}^{*}$ is a positive linear operator and also by (4)

$$
L_{n}^{*}(1 ; x)=1 .
$$

Now, we give the following Korovkin type theorem for the test functions proved by Altın, Doğru and Taşdelen [1].

Theorem A. Let $\left\{A_{n}\right\}$ be a sequence of linear positive operators from $W_{\omega}$ into $C[0, A]$ and satisfies the three conditions (7). Then for all $f \in W_{\omega}$, we have

$$
\lim _{n \rightarrow \infty}\left\|A_{n}(f)-f\right\|=0
$$

[1] .

To prove the main result for the sequence of linear positive operators $L_{n}^{*}$, we need the following two lemmas.

Lemma 1. We have 


$$
\lim _{n \rightarrow \infty}\left\|L_{n}^{*}(\theta)-\theta\right\|=0 .
$$

Proof. From (iii) and (2) we get

$$
\begin{aligned}
L_{n}^{*}(\theta ; x) & =\frac{1}{h_{n}(x, t)} \sum_{k=0}^{\infty} \frac{\Gamma_{k, n}(t)}{c_{k, n}} x^{k} \int_{a_{k, n}}^{a_{k, n}+c_{k, n}} \frac{\xi}{b_{n}} d \xi \\
& =\frac{1}{h_{n}(x, t)} \sum_{k=0}^{\infty} \frac{a_{k, n}}{b_{n}} \Gamma_{k, n}(t) x^{k}+\frac{1}{h_{n}(x, t)} \sum_{k=0}^{\infty} \frac{c_{k, n}}{2 b_{n}} \Gamma_{k, n}(t) x^{k} \\
& =L_{n}(\theta ; x)+\frac{1}{2 b_{n}} \frac{1}{h_{n}(x, t)} \sum_{k=0}^{\infty} c_{k, n} \Gamma_{k, n}(t) x^{k} .
\end{aligned}
$$

In this way, we obtain from (5)

$$
L_{n}^{*}(\theta ; x)-\theta(x)=\frac{1}{2 b_{n}} \frac{1}{h_{n}(x, t)} \sum_{k=0}^{\infty} c_{k, n} \Gamma_{k, n}(t) x^{k}
$$

Following (iii), (iv) and (9), each term of the right hand side is non- negative, we have

$$
L_{n}^{*}(\theta ; x)-\theta(x) \geq 0 .
$$

Hence from (9) and (4) we can write

$$
0 \leq L_{n}^{*}(\theta ; x)-\theta(x) \leq \frac{1}{2 b_{n}} .
$$

So we have

$$
\left\|L_{n}^{*}(\theta)-\theta\right\| \leq \frac{1}{2 b_{n}} .
$$

Taking limit for $n \rightarrow \infty$ in (14), (iii) yields that

$$
\lim _{n \rightarrow \infty}\left\|L_{n}^{*}(\theta)-\theta\right\|=0 .
$$

Lemma 2. we have

$$
\lim _{n \rightarrow \infty}\left\|L_{n}^{*}\left(\theta^{2}\right)-\theta^{2}\right\|=0
$$


Proof. From (8) and (2) we have

$$
\begin{aligned}
L_{n}^{*}\left(\theta^{2} ; x\right) & =\frac{1}{h_{n}(x, t)} \sum_{k=0}^{\infty} \frac{\Gamma_{k, n}(t)}{c_{k, n}} x^{k} \int_{a_{k, n}}^{a_{k, n}+c_{k, n}} \frac{\xi^{2}}{b_{n}^{2}} d \xi \\
& =L_{n}\left(\theta^{2} ; x\right)+\frac{1}{b_{n}} \frac{1}{h_{n}(x, t)} \sum_{k=0}^{\infty} \frac{a_{k, n}}{b_{n}} c_{k, n} \Gamma_{k, n}(t) x^{k} \\
& +\frac{1}{3 b_{n}^{2}} \frac{1}{h_{n}(x, t)} \sum_{k=0}^{\infty} c_{k, n}^{2} \Gamma_{k, n}(t) x^{k}
\end{aligned}
$$

Hence by (9) we get

$$
\begin{aligned}
L_{n}^{*}\left(\theta^{2} ; x\right)-\theta^{2}(x) & \leq L_{n}\left(\theta^{2} ; x\right)-\theta^{2}(x) \\
& +\frac{1}{b_{n}} L_{n}(\theta ; x)+\frac{1}{3 b_{n}^{2}} L_{n}(1 ; x) .
\end{aligned}
$$

So, using (4), (5) and (6) we have

$$
\begin{aligned}
L_{n}^{*}\left(\theta^{2} ; x\right)-\theta^{2}(x) & \leq \theta^{2}(x)\left(\frac{b_{n+1}}{b_{n}}-1\right) \\
& +\left(\frac{\varphi_{n}}{b_{n}}+\frac{1}{b_{n}}\right) \theta(x)+\frac{1}{3 b_{n}^{2}} .
\end{aligned}
$$

Since

$$
\left(\frac{s}{1-s}\right)^{2}=\left(\frac{s}{1-s}-\frac{x}{1-x}\right)^{2}+\frac{2 x}{1-x} \frac{s}{1-s}-\left(\frac{x}{1-x}\right)^{2}
$$

we get

$$
\begin{aligned}
L_{n}^{*}\left(\theta^{2} ; x\right)-\theta^{2}(x) & =L_{n}^{*}\left((\theta(s)-\theta(x))^{2} ; x\right) \\
& +2 \theta(x) L_{n}^{*}(\theta(s)-\theta(x) ; x) \geq 0 .
\end{aligned}
$$

Here we have also used the positivity of $L_{n}^{*}$ and (13). By taking the relations (15) and (16) into account we obtain

$$
0 \leq\left\|L_{n}^{*}\left(\theta^{2}\right)-\theta^{2}\right\| \leq B^{*}\left(\left|\frac{b_{n+1}}{b_{n}}-1\right|+\frac{\varphi_{n}+1}{b_{n}}+\frac{1}{3 b_{n}^{2}}\right),
$$

where $B^{*}=\max \left\{1, \frac{A}{1-A},\left(\frac{A}{1-A}\right)^{2}\right\}$.

Now taking limit for $n \rightarrow \infty$ in (17), (iii) and $(v)$ yield

$$
\lim _{n \rightarrow \infty}\left\|L_{n}^{*}\left(\theta^{2}\right)-\theta^{2}\right\|=0
$$


Then by using (10), Lemma 1, Lemma 2 and Theorem A we can state the following approximation theorem for the operators $L_{n}^{*}$ at once.

Theorem 1. Let $L_{n}^{*}$ be the operator given by (8). Then for all $f \in W_{w}$, we have

$$
\lim _{n \rightarrow \infty}\left\|L_{n}^{*}(f)-f\right\|=0
$$

\section{Rates of Convergence Properties}

In this section, we compute the rate of convergence of the sequence $\left\{L_{n}^{*}(f ;).\right\}$ to function $f$ by means of the modulus of continuity and the elements of modified Lipschitz class.

Theorem 2. Let $L_{n}^{*}$ be the operator given by (8). Then for all $f \in W_{\omega}$, we have

$$
\left\|L_{n}^{*}(f)-f\right\| \leq\left(1+\sqrt{B^{*}}\right) \omega\left(\delta_{n}\right)
$$

where $\delta_{n}:=\left\{\left|\frac{b_{n+1}}{b_{n}}-1\right|+\frac{\varphi_{n}+1}{b_{n}}+\frac{1}{3 b_{n}^{2}}\right\}^{\frac{1}{2}}$ and $B^{*}$ is given as in Lemma 2 .

Proof. We suppose that $f \in W_{\omega}$; then, by (10),

$$
\begin{gathered}
\left|L_{n}^{*}(f ; x)-f(x)\right| \leq \omega\left(\delta_{n}\right) L_{n}^{*}\left(\frac{|s-x|}{\delta_{n}}+1 ; x\right) \\
\leq \omega\left(\delta_{n}\right) L_{n}^{*}\left(1+\frac{1}{\delta_{n}}|\theta(s)-\theta(x)| ; x\right) \\
\left|L_{n}^{*}(f ; x)-f(x)\right|=\omega\left(\delta_{n}\right)\left[L_{n}^{*}(1 ; x)+\frac{1}{\delta_{n}} L_{n}^{*}(|\theta(s)-\theta(x)| ; x)\right] \\
=\omega\left(\delta_{n}\right)\left[1+\frac{1}{\delta_{n}} \frac{1}{h_{n}(x, t)} \sum_{k=0}^{\infty} \frac{\Gamma_{k, n}(t)}{c_{k, n}} x^{k} \int_{a_{k, n}}^{a_{k, n}+c_{k, n}}\left|\frac{\xi}{b_{n}}-\theta(x)\right| d \xi\right] .
\end{gathered}
$$

By applying the Cauchy-Schwarz inequality to the integral first and to the 
sum next, we obtain

$$
\begin{aligned}
& \left|L_{n}^{*}(f ; x)-f(x)\right| \\
\leq & \omega\left(\delta_{n}\right)\left[1+\frac{1}{\delta_{n}} \frac{1}{h_{n}(x, t)} \sum_{k=0}^{\infty} \frac{\Gamma_{k, n}(t)}{c_{k, n}} x^{k}\left(\int_{a_{k, n}}^{a_{k, n}+c_{k, n}}\left(\frac{\xi}{b_{n}}-\theta(x)\right)^{2} d \xi\right)^{\frac{1}{2}}\left(\int_{a_{k, n}}^{a_{k, n}+c_{k, n}} d \xi\right)^{\frac{1}{2}}\right] \\
\leq & \omega\left(\delta_{n}\right)\left[1+\frac{1}{\delta_{n}}\left(\frac{1}{h_{n}(x, t)} \sum_{k=0}^{\infty} \frac{\Gamma_{k, n}(t)}{c_{k, n}} x^{k} \int_{a_{k, n}}^{a_{k, n}+c_{k, n}}\left(\frac{\xi}{b_{n}}-\theta(x)\right)^{2} d \xi\right)^{\frac{1}{2}}\right. \\
& \left.\times\left(\frac{1}{h_{n}(x, t)} \sum_{k=0}^{\infty} \frac{\Gamma_{k, n}(t)}{c_{k, n}} x^{k}\right)^{\frac{1}{2}}\right] \\
\leq & \omega\left(\delta_{n}\right)\left[1+\frac{1}{\delta_{n}}\left(\frac{1}{h_{n}(x, t)} \sum_{k=0}^{\infty} \frac{\Gamma_{k, n}(t)}{c_{k, n}} x^{k} \int_{a_{k, n}}^{a_{k, n}+c_{k, n}}\left(\frac{\xi}{b_{n}}-\theta(x)\right)^{2} d \xi\right)^{\frac{1}{2}}\right] \\
\leq & \omega\left(\delta_{n}\right)\left[1+\frac{1}{\delta_{n}}\left(L_{n}^{*}\left((\theta(s)-\theta(x))^{2} ; x\right)\right)^{\frac{1}{2}}\right]
\end{aligned}
$$

This implies that

$$
\left\|L_{n}^{*}(f)-f\right\| \leq w\left(f, \delta_{n}\right)\left[1+\frac{1}{\delta_{n}}\left(\sup _{x \in[0, A]} L_{n}^{*}\left((\theta(s)-\theta(x))^{2} ; x\right)\right)^{\frac{1}{2}}\right]
$$

By using the equality (16), from (14) and (17) we get that

$$
\begin{aligned}
\left\|L_{n}^{*}\left((\theta(s)-\theta(x))^{2}\right)\right\| & \leq\left\|L_{n}^{*}\left(\theta^{2}\right)-\theta^{2}\right\| \\
& +\max _{x \in[0, A]} 2 \theta(x)\left\|\left(L_{n}^{*}(\theta)-\theta\right)\right\| \\
& \leq B^{*}\left[\left|\frac{b_{n+1}}{b_{n}}-1\right|+\frac{\varphi_{n}+1}{b_{n}}+\frac{1}{3 b_{n}^{2}}\right] .
\end{aligned}
$$

So, combining (19) with (20) we can write

$$
\left\|L_{n}^{*}(f)-f\right\| \leq \omega\left(\delta_{n}\right)\left\{1+\frac{1}{\delta_{n}}\left[B^{*}\left(\left|\frac{b_{n+1}}{b_{n}}-1\right|+\frac{\varphi_{n}+1}{b_{n}}+\frac{1}{3 b_{n}^{2}}\right)\right]^{\frac{1}{2}}\right\}
$$


For $\delta:=\delta_{n}=\left[\left|\frac{b_{n+1}}{b_{n}}-1\right|+\frac{\varphi_{n}+1}{b_{n}}+\frac{1}{3 b_{n}^{2}}\right]^{\frac{1}{2}}$ the proof is completed.

Now we will study the rate of convergence of the positive linear operators $L_{n}^{*}$ by means of the elements of the modified Lipschitz class $\widetilde{\operatorname{Lip}}_{M}(\alpha)$.

Let us consider the class of functions $f$, denoted by $\widetilde{\operatorname{Lip}}_{M}(\alpha)$, as follows:

$$
|f(s)-f(x)| \leq M|\theta(s)-\theta(x)|^{\alpha}, s, x \in[0, A], 0<A<1,
$$

where $M>0,0<\alpha \leq 1$ and $f \in C[0, A]$. We can call the class $\widetilde{\operatorname{Lip}_{M}}(\alpha)$ as "the modified Lipschitz class".

Theorem 3. Let $L_{n}^{*}$ be the operator given by (8). Then for all $f \in$ $\widetilde{\operatorname{Lip}}_{M}(\alpha)$, we have

$$
\left\|L_{n}^{*}(f)-f\right\| \leq M\left(B^{*}\right)^{\frac{\alpha}{2}} \delta_{n}^{\alpha},
$$

where $\delta_{n}$ and $B^{*}$ are the same as in Theorem 2 .

Proof. Let $f \in \widetilde{\operatorname{Lip}}_{M}(\alpha)$ and $0<\alpha \leq 1$. By linearity and monotonicity of $L_{n}^{*}$, we have

$$
\begin{aligned}
\left|L_{n}^{*}(f ; x)-f(x)\right| \leq & L_{n}^{*}(|f(s)-f(x)| ; x) \\
& \leq M L_{n}^{*}\left(|\theta(s)-\theta(x)|^{\alpha} ; x\right) \\
& =M \frac{1}{h_{n}(x, t)} \sum_{k=0}^{\infty} \frac{\Gamma_{k, n}(t)}{c_{k, n}} x^{k} \int_{a_{k, n}}^{a_{k, n}+c_{k, n}}\left|\frac{\xi}{b_{n}}-\theta(x)\right|^{\alpha} d \xi .
\end{aligned}
$$

By applying the Hölder inequality with $p=\frac{2}{\alpha}, q=\frac{2}{2-\alpha}$, to the integral first and to the sum next, then last formula is reduced to

$$
\begin{aligned}
\left|L_{n}^{*}(f ; x)-f(x)\right| & \leq M\left\{\frac{1}{h_{n}(x, t)} \sum_{k=0}^{\infty} \frac{\Gamma_{k, n}(t)}{c_{k, n}} x^{k} \int_{a_{k, n}}^{a_{k, n}+c_{k, n}}\left(\frac{\xi}{b_{n}}-\theta(x)\right)^{2} d \xi\right\}^{\frac{\alpha}{2}} \\
& \times\left\{\frac{1}{h_{n}(x, t)} \sum_{k=0}^{\infty} \Gamma_{k, n}(t) x^{k}\right\}^{\frac{2}{2-\alpha}} \\
& =M\left\{L_{n}^{*}\left((\theta(s)-\theta(x))^{2} ; x\right)\right\}^{\frac{\alpha}{2}} .
\end{aligned}
$$

By taking into account the inequality (20) in the last inequality we have

$$
\begin{aligned}
\left\|L_{n}^{*}(f)-f\right\| & \leq M\left\|L_{n}^{*}\left((\theta(s)-\theta(x))^{2}\right)\right\|^{\frac{\alpha}{2}} \\
& \leq M\left\{B^{*}\left[\left|\frac{b_{n+1}}{b_{n}}-1\right|+\frac{\varphi_{n}+1}{b_{n}}+\frac{1}{3 b_{n}^{2}}\right]\right\}^{\frac{\alpha}{2}} .
\end{aligned}
$$

Thus we obtain the desired result. 


\section{An $r$-th order generalization of the operators $L_{n}^{*}$}

By $C^{r}[0, A]\left(0<A<1, r \in N_{o}\right)$ we denote the space of all functions of having continuous $r$-th order derivative $f^{(r)}$ on the segment $[0, A],(0<A<1)$, where as usual, $f^{(0)}(x)=f(x)$.

We consider the following $r$-th order generalization of the positive linear operators $L_{n}^{*}$ defined by $(8)$ :

$$
L_{n, r}^{*}(f ; x)=\frac{1}{h_{n}(x, t)} \sum_{k=0}^{\infty} \frac{\Gamma_{k, n}(t)}{c_{k, n}} x^{k} \int_{a_{k, n}}^{a_{k, n}+c_{k, n}} \sum_{j=0}^{r} f^{(j)}\left(\frac{\xi}{\xi+b_{n}}\right) \frac{\left(x-\frac{\xi}{\xi+b_{n}}\right)^{j}}{j !} d \xi,
$$

where $f \in C^{r}[0, A] \quad\left(0<A<1, r \in \mathbb{N}_{o}\right), n \in \mathbb{N}$.

The $r$-th order generalization of the positive linear operators was given in [9]. But we remark that the $r$-th order generalization for the Kantorovichtype operators are first introduced by Özarslan, Duman and Srivastava in [15]. Note that taking $r=0$, we obtain the operators $L_{n}^{*}(f ; x)$ defined by (8).

We recall that a function $f \in[0, A]$ belongs to $\operatorname{Lip}_{M}(\alpha)$ if the following inequality holds:

$$
|f(y)-f(x)| \leq M|y-x|^{\alpha}, \quad(x, y \in[0, A]) .
$$

Theorem 4. For any $f \in C^{r}[0, A]$ such that $f^{(r)} \in \operatorname{Lip}_{M}(\alpha)$ we have

$$
\left\|L_{n, r}^{*}(f)-f\right\|_{C[0, A]} \leq \frac{M}{(r-1) !} \frac{\alpha}{\alpha+r} B(\alpha, r)\left\|L_{n}\left(|s-x|^{\alpha+r} ; x\right)\right\|_{C[0, A]},
$$

where $B(\alpha, r)$ is the beta function and $r \in \mathbb{N}_{o}, n \in \mathbb{N}$.

Proof. We can write from (21) that

$$
\begin{aligned}
f(x)-L_{n, r}^{*}(f ; x) & =\frac{1}{h_{n}(x, t)} \sum_{k=0}^{\infty} \frac{\Gamma_{k, n}(t)}{c_{k, n}} x^{k} \\
& \times \int_{a_{k, n}}^{a_{k, n}+c_{k, n}}\left[f(x)-\sum_{j=0}^{r} f^{(j)}\left(\frac{\xi}{\xi+b_{n}}\right) \frac{\left(x-\frac{\xi}{\xi+b_{n}}\right)^{j}}{j !}\right] d \xi .
\end{aligned}
$$

It is also known from Taylor's formula that

$$
\begin{aligned}
& f(x)-\sum_{j=0}^{r} f^{(j)}\left(\frac{\xi}{\xi+b_{n}}\right) \frac{\left(x-\frac{\xi}{\xi+b_{n}}\right)^{j}}{j !} \\
& =\frac{\left(x-\frac{\xi}{\xi+b_{n}}\right)^{r}}{(r-1) !} \int_{0}^{1}(1-s)^{r-1}\left[f^{(r)}\left(\frac{\xi}{\xi+b_{n}}+s\left(x-\frac{\xi}{\xi+b_{n}}\right)\right)-f^{(r)}\left(\frac{\xi}{\xi+b_{n}}\right)\right] d s .
\end{aligned}
$$


Because of $f^{(r)} \in \operatorname{Lip}_{M}(\alpha)$, one can write

$$
\left|f^{(r)}\left(\frac{\xi}{\xi+b_{n}}+s\left(x-\frac{\xi}{\xi+b_{n}}\right)\right)-f^{(r)}\left(\frac{\xi}{\xi+b_{n}}\right)\right| \leq M s^{\alpha}\left|x-\frac{\xi}{\xi+b_{n}}\right|^{\alpha} .
$$

From the well known beta function, we can write

$$
\int_{0}^{1} s^{\alpha}(1-s)^{r-1} d s=B(1+\alpha, r)=\frac{\alpha}{\alpha+r} B(\alpha, r) .
$$

Substituting (25) and (26) in (24), we conclude that

$$
\left|f(x)-\sum_{j=0}^{r} f^{(j)}\left(\frac{\xi}{\xi+b_{n}}\right) \frac{\left(x-\frac{\xi}{\xi+b_{n}}\right)^{j}}{j !}\right| \leq \frac{M}{(r-1) !} \frac{\alpha}{\alpha+r} B(\alpha, r)\left|x-\frac{\xi}{\xi+b_{n}}\right|^{\alpha+r} .
$$

By taking (23) and (27) into consideration, we arrive at (22).

Now, we consider the function $g \in C[0, A]$ defined by

$$
g(s)=|s-x|^{r+\alpha} \text {. }
$$

Since $g(x)=0$ we can write $\left\|L_{n}\left(|s-x|^{\alpha+r}\right)\right\|_{C[0, A]}=0$. Theorem 4 yields that for all $f \in C^{r}[0, A]$ such that $f^{(r)} \in \operatorname{Lip}_{M}(\alpha)$, we have

$$
\lim _{n \rightarrow \infty}\left\|L_{n}^{*[r]}(f)-f\right\|=0
$$

Finally, in view of Theorem 2, Theorem 3 and $g \in \operatorname{Lip}_{A^{r}}(\alpha)$, one can deduce the following result from Theorem 4 immediately:

Corollary 1. For all $f \in C^{r}[0, A]$, such that $f^{(r)} \in \operatorname{Lip}_{M}(\alpha)$, we have

$$
\left\|L_{n}^{*}(f)-f\right\| \leq \frac{M}{(r-1) !} \frac{\alpha}{\alpha+r} B(\alpha, r)\left(1+\sqrt{B^{*}} \omega\left(g, \delta_{n}\right)\right) .
$$

where $B^{*}$ is the same as in Lemma $2, \delta_{n}$ is the same as in Theorem 2 and $g$ is defined by (28).

Corollary 2. For all $f \in C^{r}[0, A]$ such that $f^{(r)} \in \operatorname{Lip}_{M}(\alpha)$, we have

$$
\left\|L_{n}^{[r]}(f)-f\right\| \leq \frac{M A^{r}}{(r-1) !} \frac{\alpha}{\alpha+r} B(\alpha, r)\left(B^{*}\right)^{\frac{\alpha}{2}} \delta_{n}^{\alpha}
$$

where $B^{*}$ is the same as in Lemma 2 and $\delta_{n}$ is the same as in Theorem 2 . 


\section{References}

[1] A. Altın, O. Doğru, and F. Taşdelen, The generalization of Meyer-König and Zeller operators by generating functions, J. Math. Anal. Appl. 312, 181-194 (2005).

[2] O. Agratini, Korovkin type estimates for Meyer-König and Zeller operators, Math. Ineq. Appl. no. 1, 4, 119-126 (2001).

[3] E. W. Cheney and A. Sharma, A. Bersntein power series, Canad. J. Math. 16, 241-253 (1964).

[4] O. Doğru, Approximation order and asymtotic approximation for Generalized Meyer-König and Zeller operators, Math. Balkanica, N.S. no. 3-4, 12, 359-368 (1998).

[5] O. Doğru, and N. Özalp, Approximation by Kantorovich type generalization of Meyer-König and Zeller operators, Glasnik Mat. no 36, 56 , 311-318 (2001).

[6] O. Doğru, O. Duman and C. Orhan, Statistical approximation by generalized Meyer-König and Zeller type operator, Studia Sci. Math. Hungar. no. $3,40,359-371$ (2003).

[7] A. D. Gadjiev and Ö. Çakar, On uniform approximation by Bleimann, Butzer and Hahn operators on all positive semi-axis, Trans. Acad. Sci. Azerb. Ser. Phys. Tech. Math. Sci. 19, 21-26 (1999).

[8] M. K. Khan, On the rate of convergence of Bersntein power series for functions of bounded variation, J. Approx. Theory, no. 1, 57, 90-103 (1989).

[9] G. Kirov and L. Popova, A generalization of the linear positive operators, Math. Balkanica 7, 149-162 (1993).

[10] P. P. Korovkin, Linear operators and approximation theory, Hindustan Publish Co., Delphi (1960).

[11] B. Lenze, Bersntein-Baskakov-Kantorovich operators and Lipschitz-type maximal functions, in: Approx. Th., Kecskemet, Hungary, Colloq. Math. Soc. Janos Bolyai 58, 469-496 (1990).

[12] A. Lupaş and M. W. Müller, Approximation properties of the Mnoperators, Aequationes Math. 5, 19-37 (1970).

[13] W. Meyer-König and K. Zeller, Bernsteinsche potenzreihen, Studia Math. 19, 89-94 (1960). 
[14] H. N. Mhaskar and D. V. Pai, Fundamentals of approximation theory. CRC Press, Boca Raton, FL; Narosa Publishing House, New Delhi, 2000.

[15] M. A. Özarslan, O. Duman and H. M. Srivastava, Statistical approximation results for Kantorovich type operators involving some special polynomials, Math. Comp. Mod. 48 (2008), 388-401.

[16] A. I. Stepanets, Methods of approximation theory, VSP, Leiden, 2005. ISBN: 90-6764-427-7.

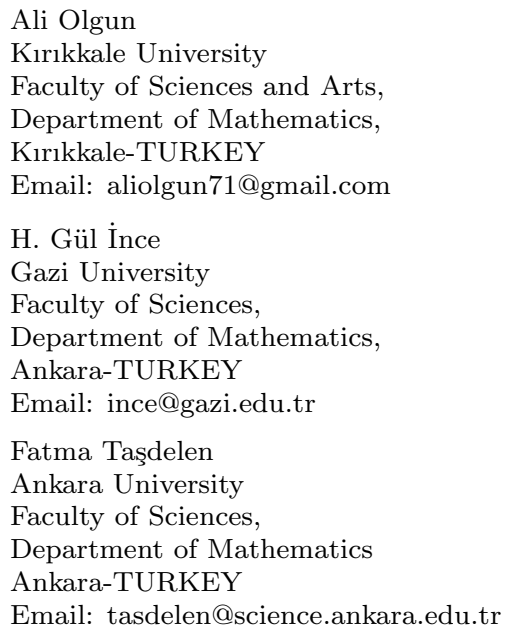


